\title{
May Measurement Month 2017-2019: A Community-Wide Opportunistic Blood Pressure Screening Campaign in Hong Kong
}

\author{
Joyce Tik Sze Li $D^{1},{ }^{1}$ Amy Shuk Man Lam $\left(D,{ }^{2}\right.$ Brian Tomlinson ${ }^{D},{ }^{3}$ \\ and Vivian Wing Yan Lee ${ }^{1}{ }^{1}$ \\ ${ }^{1}$ Centre for Learning Enhancement and Research, The Chinese University of Hong Kong, 502, Hui Yeung Shing Building, \\ Shatin, Hong Kong \\ ${ }^{2}$ Department of Medicine \& Therapeutics, Faculty of Medicine, The Chinese University of Hong Kong, Shatin, Hong Kong \\ ${ }^{3}$ Faculty of Medicine, Macau University of Science \& Technology, Block P25, Avenida Wai Long, Taipa, Macau
}

Correspondence should be addressed to Vivian Wing Yan Lee; vivianlee@cuhk.edu.hk

Received 21 September 2020; Revised 17 December 2020; Accepted 6 January 2021; Published 18 January 2021

Academic Editor: Massimo Salvetti

Copyright $(92021$ Joyce Tik Sze Li et al. This is an open access article distributed under the Creative Commons Attribution License, which permits unrestricted use, distribution, and reproduction in any medium, provided the original work is properly cited.

\begin{abstract}
Introduction. Hypertension is a modifiable risk factor for multiple cardiovascular diseases. Early identification and intervention of new cases are crucial to improve patients' outcomes. May Measurement Month (MMM) is an annual global synchronised blood pressure (BP) screening campaign. Participants can have their BP measured at the screening sites. It may be a possible way to identify undiagnosed hypertensive patients in the population. Methods. It was a cross-sectional study of BP among Hong Kong adults. Multiple screening sites were set in local community pharmacies and on the campus of the Chinese University of Hong Kong. Participants were asked to fill in a questionnaire regarding their demographics, medical history, and social history. Then, they took at least one BP reading using an automated sphygmomanometer after sitting at for 5 minutes. Up to three BP readings were taken and recorded for each participant, with one-minute intervals between readings. Results. A total of 3224 adults participated in MMM between 2017 and 2019. The average BP among the 3224 participants was 139.8/75.5 mmHg. The prevalence of hypertension was 2282 (70.8\%), of which 635 (27.8\%) were undiagnosed before MMM. Among the 1647 participants previously diagnosed with hypertension, 1007 (61.1\%) had uncontrolled hypertension. Conclusion. A high number of cases can be identified with untreated, or treated but uncontrolled, hypertension from MMM. Citizens should be encouraged to check BP regularly and take follow-up actions if hypertension is suspected.
\end{abstract}

\section{Introduction}

Hypertension is a known risk factor for multiple cardiovascular diseases and cerebrovascular events $[1,2]$. Raised blood pressure (BP) is the most important modifiable risk factor for premature death $[3,4]$. It is estimated that 1.13 billion people worldwide have hypertension, with around two-thirds living in low- and middle-income countries [5]. One of the global targets for non-communicable diseases is to reduce the prevalence of hypertension by $25 \%$ by 2025 starting from 2010 [5].

Despite the high number of cases, there are still a lot of the undiagnosed or undertreated patients. The well-known "rule of halves" in hypertension states that roughly only half of all hypertensive cases are diagnosed, half of those diagnosed are treated, and half of those treated are well controlled $[6,7]$. Locally, the Population Health Survey (PHS) 2014/15 conducted by the Department of Health revealed that the total prevalence of hypertension among persons aged 15 or above was $27.7 \%$ (25.5\% for females and $30.1 \%$ for males) [8]. Around $47.5 \%$ of them were undiagnosed before the PHS. The total prevalence of hypertension increased steadily with age from $4.5 \%$ among those aged $15-24$ to $64.8 \%$ among those aged $65-84$.

Hypertension is known as a "silent killer." While most people with hypertension are unaware of the problem because there may be no warning signs or symptoms, untreated hypertension can lead to serious complications such as 
coronary heart disease, stroke, heart failure, and renal failure [9-11]. Early identification and intervention of new cases are crucial to minimize preventable death $[12,13]$. The Hong Kong Reference Framework for Hypertension Care for Adults in Primary Care Settings has set out recommendations on the prevention and treatment of hypertension among adults [14]. It clearly states the importance of early identification of people with hypertension and recommends $\mathrm{BP}$ measurement in all adults from 18 years of age at least every 2 years.

Community-wide BP screening is a possible way to reach out citizens who do not have the habit of having a regular body check up $[15,16]$. May Measurement Month (MMM) is an annual global synchronised BP screening campaign led by the International Society of Hypertension (ISH) since May 2017 [17-19]. It aims to raise citizens' awareness on hypertension, collect the scientific evidence needed to help influence global health policy, and make BP screening more widely available around the world through MMM [20]. In 2018, 1,504,963 adults from 89 countries participated in MMM [18]. Hong Kong is one of the MMM participating places. This paper reports the findings of MMM from 2017 to 2019 in Hong Kong.

\section{Methods}

2.1. Study Design and Study Population. It was a cross-sectional opportunistic study of BP among adults in Hong Kong. The Chinese University of Hong Kong (CUHK) collaborated with two local chain community pharmacies to set up multiple BP measurement stations. Participants had their BP measured either in the community pharmacy or on the CUHK campus. Pharmacists working in the community pharmacies, as well as staff and students from the Faculty of Medicine, CUHK, helped with BP measurements. Not only did the MMM programs provide an excellent platform for primary care in the community but also for student engagement in community outreach.

Participants were recruited through convenience sampling. Participants aged 18 or above who were able to provide informed consent were recruited. The event was promoted through fliers, posters, press release, and social media. Participants were self-referred to the screening sites.

2.2. Procedures. The current study complied with the MMM BP measurement protocol set by the ISH [17-19].

Before BP measurement, the participants were seated with their backs supported and with their legs resting on the ground and in the uncrossed position for 5 minutes. Meanwhile, each participant filled in an anonymous questionnaire regarding their demographics, such as ethnicity, age, height, weight, medical history, social history, and habits of $\mathrm{BP}$ monitoring. At least one $\mathrm{BP}$ reading was taken using an Omron BP device (Omron Healthcare, Kyoto, Japan), which was an automated sphygmomanometer that measured systolic blood pressure (SBP), diastolic blood pressure (DBP), and heart rate. Upon mutual agreement with participants, three BP readings were taken and recorded, with one-minute interval between readings. In the current project, hypertension was defined as a systolic BP $\geq 140 \mathrm{mmHg}$, diastolic BP $\geq 90 \mathrm{mmHg}$, or on at least one antihypertensive medication taken for raised BP. Data collected were analysed centrally by the MMM project team.

Dietary and lifestyle information would be provided to participants found to be hypertensive. They were advised to reduce salt intake, reduce alcohol intake, engage in regular physical exercise for at least 30 minutes on most of the days of the week, have at least 5 portions of fruit and vegetables per day, and reduce weight aiming for a body mass index (BMI) target of $<25 \mathrm{~kg} / \mathrm{m}^{2}$. They were also advised to have home BP monitoring.

\section{Results}

A total of 3224 adults participated in MMM between 2017 and 2019. Since the questionnaire differed slightly between years and the data collection was incomplete for some of the items, numbers used in different analyses varied. Participant demographics are shown in Table 1. Among the 3224 participants, $22.3 \%$ were male and $77.7 \%$ were female. Their mean age was $71.9 \pm 13.0$. There were $17.7 \%$ of participants with diabetes, $9.9 \%$ with history of heart attack, and $4.6 \%$ with history of stroke. As for social history, $2.8 \%$ of participants reported tobacco use and 5.4\% reported alcohol consumption.

In total, 308 (9.6\%) participants took BP readings three times, 9 participants $(0.3 \%)$ took $\mathrm{BP}$ readings twice, and 2907 (90.2\%) took BP reading once. Of the 308 participants who took three readings, the first reading was the highest and the third reading was the lowest. Mean values of the first and third reading were $125.9 / 78.6 \mathrm{mmHg}$ and 122.3 / $76.9 \mathrm{mmHg}$, respectively.

Table 2 showed the mean BP of participants with regard to their first readings. When considering the first BP readings among the 3224 participants, their average BP was $139.8 / 75.5 \mathrm{mmHg}$. There were 1647 out of 3224 participants (51.1\%) who had been previously diagnosed with hypertension. Among the 1543 participants who had not been diagnosed with hypertension, $635(41.2 \%)$ of them were found to be hypertensive. The total number of participants with hypertension was 2282 (70.8\%). Among the 1647 participants previously diagnosed with hypertension, 1007 (61.1\%) had uncontrolled hypertension.

Tables 3 and 4 showed the mean BP of participants stratified according to medical history and social history. The mean BP of participants with diabetes, history of heart attack, and history of stroke was $141.7 / 73.1 \mathrm{mmHg}, 140.4$ / $72.7 \mathrm{mmHg}$, and $143.7 / 74.4 \mathrm{mmHg}$, respectively. The proportion of participants with hypertension was $53.2 \%, 51.1 \%$, and $59.7 \%$, respectively. The mean BP of participants who used tobacco and consumed alcohol was $130.7 / 76.6 \mathrm{mmHg}$ and $140.2 / 79.3 \mathrm{mmHg}$, respectively. The proportion of participants with hypertension was $36.8 \%$ and $51.8 \%$, respectively. 
TABle 1: Participant demographics.

\begin{tabular}{|c|c|c|c|c|}
\hline & 2017 & 2018 & 2019 & Total \\
\hline No of participants & 2358 & 717 & 149 & 3224 \\
\hline Mean age & $76.1 \pm 8.2$ & $60.3 \pm 16.0$ & $60.2 \pm 17.2$ & $71.9 \pm 13.0$ \\
\hline Mean BMI & - & $23.3 \pm 4.0$ & $23.4 \pm 5.5$ & - \\
\hline \multicolumn{5}{|l|}{ Gender } \\
\hline Male & $488 / 2358(20.7 \%)$ & $184 / 717(25.7 \%)$ & $47 / 149(31.5 \%)$ & $719 / 3224(22.3 \%)$ \\
\hline Female & $1870 / 2358(79.3 \%)$ & $533 / 717(74.3 \%)$ & $102 / 149(68.5 \%)$ & $2505 / 3224(77.7 \%)$ \\
\hline \multicolumn{5}{|l|}{ Ethnicity } \\
\hline East Asian & & $187(93.0 \%)$ & $143(96.0 \%)$ & \\
\hline South-East Asian & & $12(6.0 \%)$ & $4(2.7 \%)$ & \\
\hline White & - & $2(1.0 \%)$ & $1(0.7 \%)$ & - \\
\hline South Asian & & $0(0.0 \%)$ & $1(0.7 \%)$ & \\
\hline \multicolumn{5}{|l|}{ Medical history } \\
\hline Hypertension & $1313 / 2358(55.7 \%)$ & $277 / 683(40.6 \%)$ & $57 / 149(38.3 \%)$ & $1647 / 3190(51.6 \%)$ \\
\hline On antihypertensive medications & - & $55 / 201(27.4 \%)$ & $56 / 149(37.6 \%)$ & - \\
\hline Diabetes & $475 / 2358(20.1 \%)$ & $84 / 717(11.7 \%)$ & $12 / 149(8.1 \%)$ & $571 / 3224(17.7 \%)$ \\
\hline Previous heart attack & $289 / 2358(12.3 \%)$ & $16 / 627(2.6 \%)$ & $4 / 149(2.7 \%)$ & $309 / 3134(9.9 \%)$ \\
\hline Previous stroke & $124 / 2358(5.3 \%)$ & $17 / 627(2.7 \%)$ & $3 / 149(2.0 \%)$ & $144 / 3134(4.6 \%)$ \\
\hline \multicolumn{5}{|l|}{ Social history } \\
\hline Tobacco use & $48 / 2358(2.0 \%)$ & $30 / 631(4.8 \%)$ & 9/141 (6.4\%) & $87 / 3130(2.8 \%)$ \\
\hline Alcohol consumption & $107 / 2358(4.5 \%)$ & $47 / 628(7.5 \%)$ & $16 / 149(10.7 \%)$ & $170 / 3135(5.4 \%)$ \\
\hline
\end{tabular}

TABLE 2: Mean BP of participants using the first reading.

\begin{tabular}{|c|c|c|c|c|}
\hline & 2017 & 2018 & 2019 & Total \\
\hline Mean SBP & 142.4 & 134.6 & 126.7 & 139.8 \\
\hline Mear & 74.4 & 78.8 & 77.5 & 75.5 \\
\hline r with hypertension & $1319 / 2358(55.9 \%)$ & $292 / 717(40.7 \%)$ & $44 / 149(29.5 \%)$ & $1655 / 3224(51.3 \%)$ \\
\hline Newly found to have hypertension & $510 / 1823(28.0 \%)$ & $103 / 380(27.1 \%)$ & $22 / 79(27.8 \%)$ & $635 / 2282(27.8 \%)$ \\
\hline Uncontrolled BP with previous diagnosis of hypertension & $809 / 1313(61.6 \%)$ & $176 / 277(63.5 \%)$ & $22 / 57(38.6 \%)$ & $1007 / 1647(61.1 \%)$ \\
\hline
\end{tabular}

TABle 3: Mean BP of participants according to medical history.

\begin{tabular}{lccccc}
\hline Year & Medical history & SBP & DBP & Number with hypertension & Proportion with hypertension \\
\hline \multirow{2}{*}{2017} & Diabetes & 141.9 & 72.4 & $256 / 475$ & 53.9 \\
& Previous heart attack & 139.9 & 72.1 & $144 / 289$ & 49.8 \\
& Previous stroke & 144.3 & 74.1 & $78 / 124$ & 62.9 \\
\multirow{2}{*}{2018} & Diabetes & 142.4 & 77.2 & $43 / 84$ & 51.2 \\
& Previous heart attack & 144.3 & 81.8 & $10 / 16$ & 62.5 \\
& Previous stroke & 141.6 & 77.2 & $8 / 17$ & 47.1 \\
\hline \multirow{2}{*}{2019} & Diabetes & 129.3 & 72.7 & $5 / 12$ & 41.7 \\
& Previous heart attack & 158.5 & 78.5 & $4 / 4$ & 100 \\
\hline \multirow{2}{*}{ Total } & Previous stroke & 128.0 & 72.7 & $0 / 3$ & 0 \\
& Diabetes & 141.7 & 73.1 & $304 / 571$ & 53.2 \\
& Previous heart attack & 140.4 & 72.7 & $158 / 309$ & 51.1 \\
& Previous stroke & 143.7 & 74.4 & $56 / 144$ & 5.7 \\
\hline
\end{tabular}

\section{Discussion}

May Measurement Month is a community-wide opportunistic hypertension screening programme. Participants volunteer to have their BP measured at the screening sites. The results were based on one single set of readings. Numerous factors such as noise from the surrounding, exercise, nicotine or caffeine intake prior to BP measurement, and white coat hypertension could affect the accuracy of measurements [21-23]. Thus, participants were informed that the results should not be treated as a diagnosis. In order to increase the reliability of BP measurements, MMM suggested all participants to take three readings and calculate the mean of the second and third reading for interpretation. The MMM BP measurement protocol was similar to that recommended by the World Health Organisation (WHO) [24]. Nevertheless, it was not practical in some of the screening sites in Hong Kong due to multiple reasons, such as lack of space for participants to sit and rest in the community pharmacies, inadequate manpower in the 
TABLE 4: Mean BP of participants according to social history.

\begin{tabular}{lccccc}
\hline Year & Social history & SBP & DBP & Number with hypertension & Proportion with hypertension (\%) \\
\hline \multirow{2}{*}{2017} & Tobacco use & 135.8 & 75.7 & $21 / 48$ & 43.8 \\
& Alcohol consumption & 142.6 & 77.1 & $60 / 107$ & 56.1 \\
\hline \multirow{2}{*}{2018} & Tobacco use & 124.1 & 77.1 & $9 / 30$ & 30.0 \\
& Alcohol consumption & 138.0 & 83.7 & $22 / 47$ & 46.8 \\
\multirow{2}{*}{2019} & Tobacco use & 126.0 & 79.2 & $2 / 9$ & 22.2 \\
& Alcohol consumption & 130.8 & 81.4 & $6 / 16$ & 37.5 \\
\multirow{2}{*}{ Total } & Tobacco use & 130.7 & 76.6 & $32 / 87$ & 36.8 \\
& Alcohol consumption & 140.2 & 79.3 & $88 / 170$ & 51.8 \\
\hline
\end{tabular}

pharmacy counters, and participants had hectic schedules and were not eager to take three readings. Consequently, data interpretation was mainly based on the first readings.

Combining the results form MMM17, MMM18, and MMM19, $70.8 \%$ of participants were found to be hypertensive, of which $27.8 \%$ were undiagnosed before MMM. This number was comparable to that reported in PHS 2014/ 15 [8]. PHS 2014/15 revealed that, among the $27.7 \%$ of citizens aged 15 or above found to have hypertension, $47.7 \%$ of them were undiagnosed before PHS. In the subgroup of citizens aged $65-84,64.8 \%$ were found to have hypertension and $20.9 \%$ of them were undiagnosed before PHS. The mean age of MMM participants was around 72 years, and the results were similar to that found in the subgroup of PHS participants aged 65-84. Therefore, although MMM provided $\mathrm{BP}$ screening service for all adults, the findings between 2017 and 2019 might be more representative for the older population.

Among the 1,508,130 participants in MMM19 worldwide, $34.9 \%$ were found to have hypertension [19]. There were $18.6 \%$ of participants who were not taking antihypertensive medications found to have hypertension. Among those already taking antihypertensive medications, 38.3\% had uncontrolled hypertension. Among the 280,863 participants from East Asia, 30.6\% had hypertension. There were $14.8 \%$ of participants who were not taking antihypertensive medications found to have hypertension and $33.4 \%$ of participants taking antihypertensive found to have uncontrolled hypertension.

The proportion of Hong Kong participants with hypertension appeared to be higher than the average results worldwide, as well as in East Asia. Nevertheless, the numbers presented by the MMM project team were analysed using the mean BP from the second and third readings. The proportions were also calculated after imputation and standardization for age and sex according to the WHO worldstandard population. Direct comparison between numbers was not feasible. However, it was clear that the proportion of participants newly found to have hypertension was high, which indicated that community-wide screening might be useful to identify new cases. It was essential for participants to take follow-up actions so as to confirm the diagnosis and receive nonpharmacological or pharmacological interventions when indicated. The risk of cardiovascular diseases could only be reduced if participants were motivated and willing to take further actions.
Participants in MMM were probably aware of the importance of BP monitoring. In 2018, there were 187 out of 192 (97.4\%) participants who had measured BP before MMM. Among them, 149 out of 187 (79.7\%) had measured $\mathrm{BP}$ within the previous 12 months. It was understandable as those who volunteered to participate were expected to be more attentive to own health. Participants also appeared to have healthier lifestyle as compared to the whole Hong Kong population. There were $2.8 \%$ of participants who smoked and 5.4\% who consumed alcohol. These proportions were lower than reported in Health Behaviour Survey 2018/19, which stated that $17.9 \%$ of the population aged 15 or above had even smoked and $8.8 \%$ of the population drank alcohol at least once a week [25]. Nevertheless, the proportion of participants with uncontrolled hypertension was high. Among those previously diagnosed with hypertension, $61.1 \%$ had uncontrolled hypertension. MMM could be a platform to advocate ambulatory BP monitoring and encourage participants to measure BP regularly.

The purpose of screening is to promote individual health through early detection of diseases [26]. It is particularly important if disease prognosis can be improved significantly by early treatment [27]. However, mass screening can be costly, especially when the equipment needed is expensive or a specific technique is needed during the collection process [27]. The screening should also be tailored to the characteristics of the population [28]. Thus, it is essential to select targeted diseases and screening tests for mass screening. On the other hand, community health screening would be less effective if the participation rate is low. Unlike schoolchildren who may have health check during periodic medical visits, screening among adults is often opportunistic [26]. Previous studies have identified the barriers and facilitators to health screening among adults [29]. Some common barriers include fear of getting disease and consequence, low risk perception, painful and uncomfortable screening procedure, lack of time, and embarrassing screening procedure [30-33]. From the experience in MMM, BP screening was easy to perform. The automated sphygmomanometers were reusable, so the equipment cost was low. The measurement processes were quick, and no uncomfortable or embarrassing procedures were involved. Participants acceptance would be higher. Yet, it successfully identified new hypertension cases, which might reduce the risk of complications and comorbidities. Therefore, we believe community-wide BP screening should be encouraged. 
Advancement in technology has made ambulatory and continuous health monitoring feasible. Consumer-grade digital devices such as smart watches and mobile applications allow self-monitoring of personal activity [34]. They can provide feedbacks and analyses to users based on activity measures. Data captured can facilitate communication with healthcare providers and family members. These devices can benefit treatment and research in multiple areas, such as seizure detection, heart rate monitoring, diabetes selfmanagement, and detection of emotional states [35-38]. With the help of smart watches, citizens can have their BP measured at rest, during daily activities, and during exercise. As the number of smart watch users grows, the amount of data collected is expected to increase as well. It may be an alternative means of screening. Still, postdiagnosis intervention is essential to promote patients' outcome. Users should be encouraged to adopt lifestyle modification and seek medical attention if persistently elevated BP is detected. It will require interprofessional collaboration to develop a system that provides holistic care to citizens, including BP measurement, postscreening counselling, and data processing.

A major aim of MMM is to raise people's awareness on hypertension [20]. The screenings were convenient, timely, and free of charge which attracted volunteers to participate. PHS 2014/15 reported that $41.2 \%$ of citizens aged $65-74$ had the habit of regular medical checkup [8]. While regular body checkup is essential, frequent self-monitoring of health parameters could also improve disease control [39-43]. Due to the COVID-19 pandemic, a lot of the community health screening activities planned in 2020 have been cancelled, including MMM20. Community centres are temporarily closed; thus, members are unable to have regular BP measurement there. While it is hoped that health screening can be resumed as soon as the outbreak dies down, patient empowerment is also critical. It is crucial to educate and provide support to citizens so that they can self-monitor BP. Besides, lifestyle changes during the COVID-19 outbreak such as reduced physical activities, reduced exercise level, and increased consumption of preserved food can hamper patients' BP control [44-47]. It is essential for citizens to recognise the long-term impact of raised $\mathrm{BP}$ and increase control over their own health.

The present study has a few limitations. Firstly, the number of participants in MMM17, MMM18, and MMM19 were different. Factors such as promotion strategies, number and location of screening sites, and number of helpers recruited might have affected the scale of event. In addition, due to the social movement in 2019 , some of the screening sites were closed. The number of participants in MMM19 was greatly reduced. Thus, it was hard to compare the results across years. Secondly, the proportion of female participants was higher than that of male, which implied a possibility of selection bias. This phenomenon correlated with the pattern observed in other public screening or health outreach activities in Hong Kong [48-50]. Some previous studies also revealed gender differences in primary care consultation rates $[51,52]$. It was suggested that female citizens might be more motivated to participate in health screening activities.
Thirdly, the sample size in some of the subgroups was small, which makes the results less representative. Some participants did not complete the whole questionnaire, and we could not assign them to any subgroups. Also, the questionnaire was modified each year and some questions were newly added. Therefore, the number of responders in those questions was fewer.

\section{Conclusions}

The results from MMM reveal that high numbers of people can be identified with untreated, or treated but uncontrolled, hypertension from an opportunistic screening campaign. Health screening should be hosted regularly to identify new cases and raise citizens' awareness on hypertension. Citizens should be encouraged to self-monitor BP and take follow-up actions if hypertension is suspected.

\section{Abbreviations \\ BP: $\quad$ Blood pressure \\ CUHK: The Chinese University of Hong Kong \\ DBP: Diastolic blood pressure \\ MMM: May Measurement Month \\ PHS: Population health survey \\ SBP: $\quad$ Systolic blood pressure \\ WHO: World Health Organisation.}

\section{Data Availability}

The datasets used and analysed during the current study are available from the corresponding author on reasonable request.

\section{Ethical Approval}

The current study has been approved by the Joint Chinese University of Hong Kong- New Territories East Cluster Clinical Research Ethics Committee, with CREC Ref No. 2017.246.

\section{Conflicts of Interest}

All authors have disclosed no conflicts of interest.

\section{Authors' Contributions}

Li JTS was responsible for drafting the manuscript, Lam ASM was responsible for data collection and analysis, Tomlinson B contributed to the study design, and Lee VWY performed critical revision for important intellectual content.

\section{Acknowledgments}

The authors thank the staff and students form the Faculty of Medicine, CUHK, and the pharmacists from Mannings and Watsons Hong Kong for helping with BP measurement. 


\section{References}

[1] M. H. Forouzanfar, P. Liu, G. A. Roth et al., "Global burden of hypertension and systolic blood pressure of at least 110 to 115 mm Hg, 1990-2015," JAMA, vol. 317, no. 2, pp. 165-182, 2017.

[2] GBD 2016 Risk Factors Collaborators, "Global, regional, and national comparative risk assessment of 84 behavioural, environmental and occupational, and metabolic risks or clusters of risks, 1990-2016: a systematic analysis for the Global Burden of Disease Study 2016," Lancet, vol. 390, no. 10100, pp. 1345-1422, 2017.

[3] A. J. Adler, D. Prabhakaran, P. Bovet et al., "Reducing cardiovascular mortality through prevention and management of raised blood pressure: a world heart federation roadmap," Global Heart, vol. 10, no. 2, pp. 111-122, 2015.

[4] P. M. Kearney, M. Whelton, K. Reynolds, P. Muntner, P. K. Whelton, and J. He, "Global burden of hypertension: analysis of worldwide data," The Lancet, vol. 365, no. 9455, pp. 217-223, 2005.

[5] WHO, Hypertension, World Health Organisation, Geneva, Switzerland, 2019.

[6] R. Hadaye, V. Kale, and R. M. Manapurath, "Strategic implications of changing rule of halves in hypertension: a crosssectional observational study," Journal of Family Medicine and Primary Care, vol. 8, no. 3, pp. 1049-1053, 2019.

[7] R. Deepa, C. S. Shanthirani, R. Pradeepa, and V. Mohan, "Is the "rule of halves" in hypertension still valid?-Evidence from the Chennai Urban Population Study," Jounal of Associations of Physicians India, vol. 51, pp. 153-157, 2003.

[8] Centre for Health Protection, Report of Population Health Survey 2014/2015. Surveillance and Epidemiology Branch, Centre for Health Protection, HongKong, 2017.

[9] L. M. Prisant, "Hypertensive heart disease," Journa of Clinical Hypertension (Greenwich), vol. 7, no. 4, pp. 231-238, 2005.

[10] Y. Kokubo and C. Matsumoto, "Hypertension is a risk factor for several types of heart disease: review of prospective studies," Advances in Experimental Medicine and Biology, vol. 956, pp. 419-426, 2017.

[11] M. H. Crawford, "Foreword," Cardiology Clinics, vol. 28, no. 4, p. xi, 2010.

[12] R. M. Carey, P. Muntner, H. B. Bosworth, and P. K. Whelton, "Prevention and control of hypertension," Journal of the American College of Cardiology, vol. 72, no. 11, pp. 1278-1293, 2018.

[13] C. Sierra and A. de la Sierra, "Early detection and management of the high-risk patient with elevated blood pressure," Vascular Health and Risk Management, vol. 4, no. 2, pp. 289-296, 2008.

[14] Food and Health Bureau, Hong Kong reference Framework for hypertension care for adults in primary care settings, Food and Health Bureau, Hong Kong, 2018.

[15] W. Maimaris, J. Paty, P. Perel et al., “The influence of health systems on hypertension awareness, treatment, and control: a systematic literature review," PloS $\mathrm{Med}$, vol. 10, no. 7, p. e1001490, 2013.

[16] J. Maurer and A. Ramos, "One-year routine opportunistic screening for hypertension in formal medical settings and potential improvements in hypertension awareness among older persons in developing countries: evidence from the Study on Global Ageing and Adult Health (SAGE)," American Journal of Epidemiology, vol. 181, no. 3, pp. 180-184, 2015.

[17] T. Beaney, A. E. Schutte, M. Tomaszewski et al., "May Measurement Month 2017: an analysis of blood pressure screening results worldwide," Lancet Glob Health, vol. 6, no. 7, pp. e736-e743, 2018.

[18] T. Beaney, L. M. Burrell, R. R. Castillo et al., "May Measurement Month 2018: a pragmatic global screening campaign to raise awareness of blood pressure by the International Society of Hypertension," European Heart Journal, vol. 40, no. 25, pp. 2006-2017, 2019.

[19] T. Beaney, A. E. Schutte, G. S. Stergiou et al., "May measurement month 2019," Hypertension, vol. 76, no. 2, pp. 333-341, 2020.

[20] N. R. Poulter and D. T. Lackland, "May Measurement Month: a global blood pressure screening campaign," The Lancet, vol. 389, no. 10080, pp. 1678-1680, 2017.

[21] G. Parati, G. Stergiou, E. O’Brien et al., "European Society of Hypertension practice guidelines for ambulatory blood pressure monitoring," Journal of Hypertension, vol. 32, no. 7, pp. 1359-1366, 2014.

[22] B. Williams, G. Mancia, W. Spiering et al., "ESC/ESH Guidelines for the management of arterial hypertension," European Heart Journal, vol. 39, no. 33, pp. 3021-3104, 2018.

[23] P. K. Whelton, R. M. Carey, W. S. Aronow et al., "Guideline for the prevention, detection, evaluation, and management of high blood pressure in adults: executive summary: a report of the American college of cardiology/American heart association task force on clinical practice guidelines," Circulation, vol. 138, no. 17, pp. e426-e483, 2017.

[24] WHO, WHO Technical Specifications for Automated Noninvasive Blood Pressure Measuring Devices with Cuff, World Health Organization, Geneva, Switzerland, Licence: CC BYNC-SA 3.0 IGO, 2020.

[25] Centre for Health Protection, Report of Health Behaviour Survey 2018/19. Non-communicable Disease Branch, Centre for Health Protection, Department of Health, Hongkong, 2020.

[26] M. Hsairi, F. Mehdi, R. Bellaaj, and M. Kassis, "Health screening strategies in Maghreb countries: situation analysis and perspectives," La Tunisie Medicale, vol. 96, no. 10-11, pp. 688-695, 2018.

[27] C. Krauth, "Health economic analysis of screening," GMS Current Topics in Otorhinolaryngology, Head and Neck Surgery, vol. 7, p. Doc01, 2008.

[28] K. A. Foley, J. Shelton, E. Richardson et al., "Primary care women's health screening: a case study of a community engaged human centered design approach to enhancing the screening process," Maternal and Child Health Journal, vol. 23, no. 11, pp. 1446-1458, 2019.

[29] C. H. Teo, C. J. Ng, A. Booth, and A. White, "Barriers and facilitators to health screening in men: a systematic review," Social Science \& Medicine, vol. 165, pp. 168-176, 2016.

[30] C. M. Borkhoff, R. Saskin, L. Rabeneck et al., "Disparities in receipt of screening tests for cancer, diabetes and high cholesterol in Ontario, Canada: a population-based study using area-based methods," Canadian Journal of Public Health, vol. 104, no. 4, pp. e284-e290, 2013.

[31] O. Garcia-Dominic, E. J. Lengerich, L. A. Wray et al., "Barriers to CRC screening among Latino adults in Pennsylvania: ACCN results," American Journal of Health Behavior, vol. 36, no. 2, pp. 153-167, 2012.

[32] H. Lee, J. Cho, D. W. Shin et al., "Association of cardiovascular health screening with mortality, clinical outcomes, and health care cost: a nationwide cohort study," Preventive Medicine, vol. 70, pp. 19-25, 2015.

[33] S. H. Lo, J. Waller, J. Wardle, and C. von Wagner, "Comparing barriers to colorectal cancer screening with barriers to breast 
and cervical screening: a population-based survey of screening-age women in Great Britain," Journal of Medical Screening, vol. 20, no. 2, pp. 73-79, 2013.

[34] B. Reeder and A. David, "Health at hand: a systematic review of smart watch uses for health and wellness," Journal of Biomedical Informatics, vol. 63, pp. 269-276, 2016.

[35] J. Lockman, R. S. Fisher, and D. M. Olson, "Detection of seizure-like movements using a wrist accelerometer," Epilepsy \& Behavior, vol. 20, no. 4, pp. 638-641, 2011.

[36] E. Jovanov, "Preliminary analysis of the use of smartwatches for longitudinal health monitoring," in Proceedings of Conference Proceedings IEEE Engineering in Medical and Biology Society, pp. 865-868, Milan, Italy, August 2015.

[37] E. Årsand, M. Muzny, M. Bradway, J. Muzik, and G. Hartvigsen, "Performance of the first combined smartwatch and smartphone diabetes diary application study," Journal of Diabetes Science and Technology, vol. 9, no. 3, pp. 556-563, 2015.

[38] M. R. Kamdar and M. J. Wu, "PRISM: a data-driven platform for monitoring mental health," Pacific Symposium on Biocomputing. Pacific Symposium on Biocomputing, vol. 21, pp. 333-344, 2016.

[39] M. W. Huygens, I. C. Swinkels, J. D. de Jong et al., "Selfmonitoring of health data by patients with a chronic disease: does disease controllability matter?" BMC Family Practice, vol. 18 , no. 1 , p. $40,2017$.

[40] N. S. Jesus Jr, A. N. Nemencio Jr, K. M. Tan, and M. B. Grava, "Self-monitoring of blood glucose as part of a multi-component therapy among non-insulin requiring type 2 diabetes patients: a meta-analysis (1966-2004)," Current Medical Research and Opinion, vol. 21, no. 2, pp. 173-183, 2005.

[41] M. L. Butryn, S. Phelan, J. O. Hill, and R. R. Wing, "Consistent self-monitoring of weight: a key component of successful weight loss maintenance**," Obesity, vol. 15, no. 12, pp. 3091-3096, 2007.

[42] A. J. Karter, L. M. Ackerson, J. A. Darbinian et al., "Selfmonitoring of blood glucose levels and glycemic control: the Northern California Kaiser Permanente Diabetes registry*," The American Journal of Medicine, vol. 111, no. 1, pp. 1-9, 2001.

[43] L. Di Renzo, P. Gualtieri, F. Pivari et al., "Eating habits and lifestyle changes during COVID-19 lockdown: an Italian survey," Journal of Translational Medicine, vol. 18, no. 1, p. 229, 2020.

[44] V. Balanzá-Martínez, B. Atienza-Carbonell, F. Kapczinski, and R. B. De Boni, "Lifestyle behaviours during the COVID19-time to connect," Acta Psychiatrica Scandinavica, vol. 141, no. 5, pp. 399-400, 2020.

[45] A. Pietrobelli, L. Pecoraro, A. Ferruzzi et al., "Effects of COVID-19 Lockdown on Lifestyle Behaviors in Children with Obesity Living in Verona, Italy: A Longitudinal Study," Obesity (Silver Spring), vol. 28, no. 8, pp. 1382-1385, 2020.

[46] M. Hamer, M. Kivimäki, C. R. Gale, and G. D. Batty, "Lifestyle risk factors, inflammatory mechanisms, and COVID-19 hospitalization: a community-based cohort study of 387,109 adults in UK," Brain, Behavior, and Immunity, vol. 87, pp. 184-187, 2020.

[47] F. Magro, C. Abreu, and J.-F. Rahier, "The daily impact of COVID-19 in gastroenterology," United European Gastroenterology Journal, vol. 8, no. 5, pp. 520-527, 2020.

[48] V. W. Y. Lee, F. Y. H. Fong, F. W. T. Cheng, and B. P. Y. Yan, "Life's simple 7 and cardiovascular disease risk knowledge in Hong Kong," BMC Cardiovascular Disorders, vol. 19, no. 1, p. 185, 2019.
[49] N.-y. Chan and C.-c. Choy, "Screening for atrial fibrillation in 13122 Hong Kong citizens with smartphone electrocardiogram," Heart, vol. 103, no. 1, pp. 24-31, 2017.

[50] P. K.-T. Li, B. C.-H. Kwan, C. B. Leung et al., "Prevalence of silent kidney disease in Hong Kong: the screening for Hong Kong Asymptomatic Renal Population and Evaluation (SHARE) program," Kidney International, vol. 67, no. 94, pp. S36-S40, 2005.

[51] Y. Wang, K. Hunt, I. Nazareth, N. Freemantle, and I. Petersen, "Do men consult less than women? An analysis of routinely collected UK general practice data," BMJ Open, vol. 3, no. 8, p. e003320, 2013.

[52] E. Tsukishima, K. Takahashi, K. Yano, and M. Mori, "Differences in factors associated with health checkup participation between persons with differing income levels. A crosssectional analysis using residential taxation as a measure of household income," Nihon Koshu Eisei Zasshi, vol. 59, no. 11, pp. 810-821, 2012. 